\title{
Resolving splits in location/allocation modeling: a heuristic procedure for transit center decisions
}

\author{
Keith A. Willoughby ${ }^{a, *}$, Dean H. Uyeno ${ }^{b}$ \\ ${ }^{a}$ Department of Management, Bucknell University, Lewisburg, PA 17837, USA \\ ${ }^{\mathrm{b}}$ Faculty of Medicine, University of British Columbia, Vancouver, BC, Canada V6T $1 Z 3$ \\ Received 5 October 1999; received in revised form 18 April 2000; accepted 26 April 2000
}

\begin{abstract}
A heuristic procedure is developed to assign buses to transit centers (garages) in such a way that all the buses on a particular route are assigned to a single transit center. This research builds on an optimal mixed integer programming location/allocation model that splits the bus assignments when capacity limitations were reached at a transit center. The heuristic procedure adopts a two-step process: namely, assignment of all buses of a route to a unique transit center, then switching of routes to alternative transit centers to enforce capacity limitations. The procedure is shown to still provide cost savings over current locations and allocations for the Vancouver Regional Transit System (VRTS), Canada's largest urban transit network. (C) 2000 Elsevier Science Ltd. All rights reserved.
\end{abstract}

Keywords: Heuristic procedure; Location modeling

\section{Introduction}

Transit systems represent a fruitful area for transportation research. Transit fare setting, transit planning, the analysis and estimation of ridership markets, and transit scheduling illustrate the assortment of issues examined. Various analytical techniques have been utilized, such as integer programming, simulation and goal programming.

The determination of the optimal number, location and size of transit centers (also referred to as bus depots or bus garages) to serve an existing (or planned) network of transit routes has also been examined. However, due to transit center capacity limitations, the solutions provided by analytical methods such as we have used in the past may "split" the buses of a given route between

\footnotetext{
${ }^{*}$ Corresponding author. Tel.: +1-570-577-1306; fax: +1-570-577-1338.

E-mail address: kwilloug@bucknell.edu (K.A. Willoughby).
} 
two or more garages. This presents operational difficulties for scheduling personnel. Can one develop a solution that allocates all buses of a specific route to a single transit center, obeys capacity restrictions, and still is relatively cost-effective? The purpose of this paper is to present a heuristic procedure aimed at doing just that.

Section 2 offers a review of literature pertinent to this paper, while Section 3 discusses the original mixed integer programming (MIP) model used to solve the transit center location problem. Section 4 describes the heuristic procedure developed to resolve any split allocations, while Section 5 illustrates the results of the procedure for various MIP model runs. Summary and conclusions are offered in Section 6.

\section{Literature review}

The determination of the optimal number, size and location of "facilities" to serve a base of "customers" is one of a class of problems known as location/allocation problems. Research in this area has been extensive: Cooper (1963) was an early contributor. An assortment of applications has been examined: warehouses, audit offices and ambulance centers represent but a few (see Love et al., 1988). The papers discussed herein are only those most directly relevant to the issue at hand.

Maze et al. (1982, 1983) created a MIP formulation for the transit center problem. In their model, they attempted to minimize vehicle deadhead (the non-revenue transportation time incurred in pulling a bus into or out of service) and driver relief (the costs associated with relieving drivers, when vehicle service schedules extended longer than the maximum driver time stipulated by union contract). They also considered the operating and capital costs of transit facilities.

The computer technology of the day did not permit the solution of their large-scale problem as originally formulated. As a result, they removed the fixed costs of opening/not opening a transit center site from the model. This reduction in 0-1 variables allowed the problem to be solved as a series of transportation problems, a type of problem that has a naturally occurring integer solution. Application of Khumawala's (1974) delta and omega heuristic procedures were then used to compare the incremental variable transportation costs against the fixed cost of constructing a transit center.

A major difficulty with linear transportation algorithms is that they can yield optimal solutions, which assign portions of the activity in one area to more than one territory. In terms of this paper, the transit routes constitute the activity of the "area", while transit centers represent respective "territories". Although Maze et al. $(1982,1983)$ were able to apply their methodology to a transit system in Detroit, Michigan (over 1000 buses), their method did not force the assignment of all buses in a given route to the same transit center.

The problem of grouping small geographic units or areas into larger geographic clusters is referred to as "districting". One key feature of the final solution in these types of problems is that an area may not be split between different territories (the so-called single source condition). Thus, approaches developed to solve these districting problems ought to provide some relevance to the current transit center issue.

Researchers have developed a variety of methods to resolve splits. Hess and Samuels (1971) applied linear programming (LP) to the assignment of geographical units to sales territories. 
A geographical unit, split among multiple territories, was assigned to the territory which received the largest proportion of the area in the original LP.

Fleischmann and Paraschis (1988) described the case of a German consumer goods manufacturer which wanted to group over 1400 geographical areas into territories of roughly equal size for its 168 sales agents. To provide such a solution, territories were forced to fall within upper and lower size limits. The authors developed a local assignment heuristic procedure to resolve splits by proceeding through a number of partial or full assignments of split areas to territories. A full assignment, for example, would occur if the size of a specific territory without split areas plus the concomitant activity of a new area still rendered the solution feasible (the solution does not violate the territorial size limits). The authors found that this procedure performed well. The remaining manual work to resolve any split areas not allocated to territories in the procedure was extremely light.

Hojati (1996) assigned census tracts to political districts, amalgamating split areas through a sequence of capacitated transportation problems. Arcs connecting split areas to adjacent territories were either given infinite capacity, or a capacity of zero in the stages of his heuristic procedure. Using 1993 data from the city of Saskatoon (Canada), he was able to assign the 42 census tracts into 11 constituencies. The political districts were more compact than the currently adopted assignment and the number of split areas was reduced.

\section{Transit center location formulation}

The Vancouver Regional Transit System (VRTS), owned and operated by BC Transit (a provincially owned Crown Corporation), serves the largest transit service area in Canada, covering an area of roughly $1800 \mathrm{~km}^{2}$. Its system includes over 900 buses (250 electrically powered trolleys and over 650 diesel buses) as well as a ferry system (SeaBus) and light rapid transit (SkyTrain). The SeaBus, the first marine transit service of its kind in the world, connects the cities of North Vancouver and Vancouver, carrying about two million passengers per year. The $S k y$ Train system, the world's longest completely automated, driverless rapid transit system, began construction in 1985. It now links Vancouver with several eastern suburbs.

The VRTS currently has five transit centers (garages) for buses. BC Transit staff identified five additional locations for transit centers (these are termed as "candidate" facilities). Table 1 lists each site, its current capacity and allocation (for existing transit centers), the minimum and maximum capacities used in the mathematical program and whether or not the transit center can house trolley buses. Sites are indicated with an "E" or " $C$ " to denote existing or candidate transit centers, respectively. Fig. 1 illustrates the location of the 10 transit centers, as well as other prominent transit features in the system.

In order to determine the optimal number, location and size of transit centers to serve the route network of the VRTS, a MIP model was constructed. It is as follows (detailed information regarding such issues as parameter estimation and data collection can be obtained in Willoughby (1993) and Uyeno and Willoughby (1995)):

$$
\text { Minimize total cost }=\sum_{r} \sum_{d} \sum_{p} \sum_{s} C_{r d p s} X_{r d p s}+\sum_{s=\text { candidate }} V_{s} N_{s}+\sum_{s=\mathrm{BTC}} F_{s} W_{s}-\sum_{s=\text { existing }} R_{s} Z_{s},
$$


Table 1

Existing and candidate transit centre locations

\begin{tabular}{lllllll}
\hline $\begin{array}{l}\text { Site } \\
\text { number }\end{array}$ & Location & $\begin{array}{l}\text { Current } \\
\text { allocation }\end{array}$ & $\begin{array}{l}\text { Current } \\
\text { capacity }\end{array}$ & $\begin{array}{l}\text { Minimum } \\
\text { size }\end{array}$ & $\begin{array}{l}\text { Maximum } \\
\text { size }\end{array}$ & $\begin{array}{l}\text { Trolley } \\
\text { accessible }\end{array}$ \\
\hline E1 & North Vancouver & 78 & 60 & 50 & 60 & \\
E2 & Port Coquitlam & 114 & 250 & 50 & 250 & \\
E3 & Surrey & 132 & 250 & 50 & 250 & Yes \\
E4 & Burnaby & 166 & 160 & 50 & 160 & Yes \\
E5 & Oakridge & 410 & 350 & 50 & 350 & \\
C1 & BCRTC & - & & 50 & 250 & \\
C2 & Cloverdale & - & 50 & 250 & \\
C3 & Lougheed Park \& & - & 50 & 250 & \\
& Ride & & & 50 & 250 & \\
C4 & Richmond & - & & 50 & 250 & Yes \\
C5 & Main \& Terminal & - & & & & \\
\hline
\end{tabular}

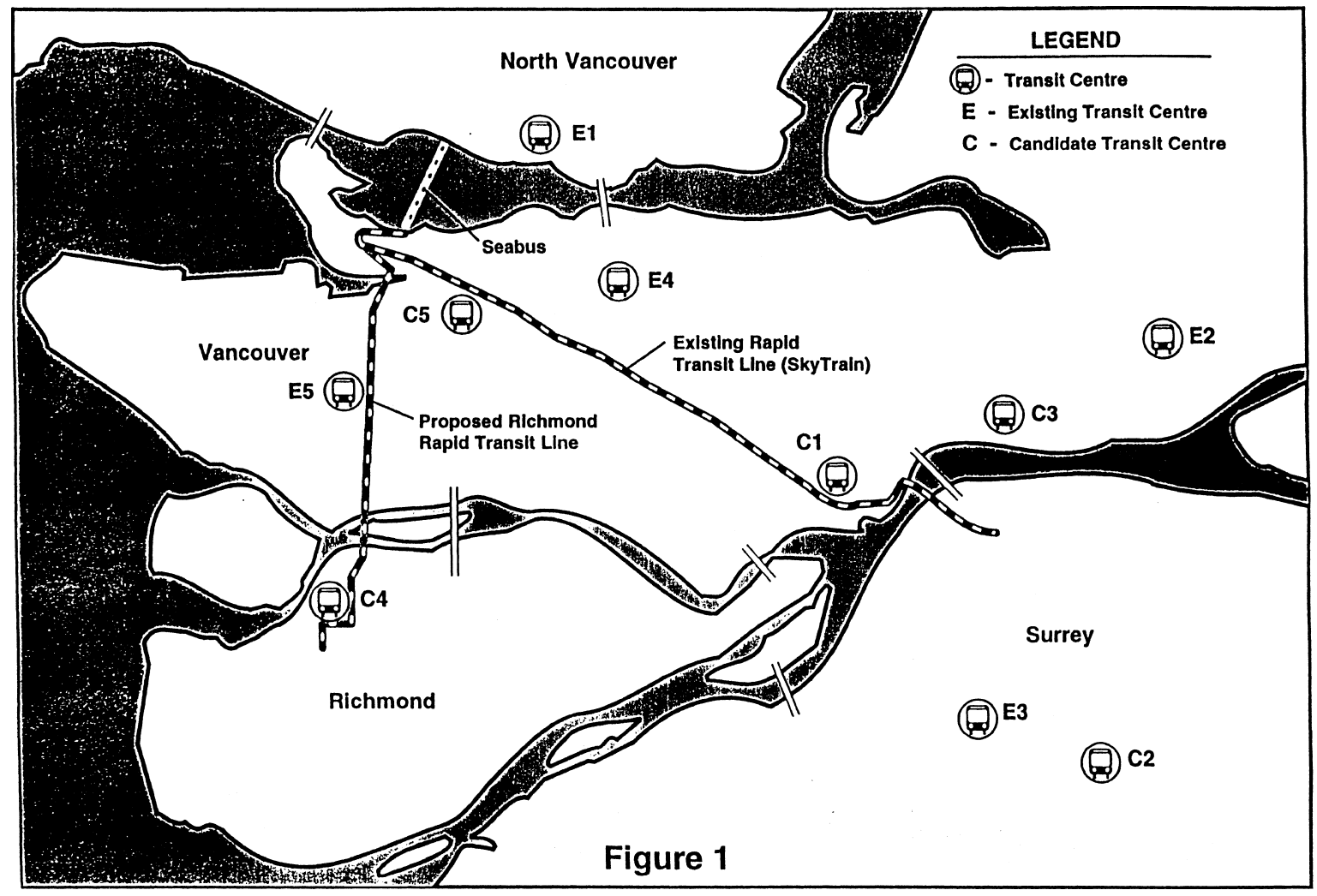

Fig. 1. Location of key transit facilities. 
subject to

$$
\begin{aligned}
& \sum_{s} X_{r d p s}=D_{r d p} \quad \forall r, d, p, \\
& \sum_{r} \sum_{d} \sum_{p} X_{r d p s}-A_{s} \leqslant 0 \quad \forall s, \\
& N_{s}-\alpha A_{s} \geqslant 0 \quad \forall s, \\
& N_{s}-\omega_{s} Y_{s} \leqslant 0 \quad \forall s=\text { candidate }, \\
& N_{s}-\lambda_{s} Y_{s} \geqslant 0 \quad \forall s=\text { candidate }, \\
& N_{s}-\beta_{s}\left(1-Z_{s}\right) \leqslant 0 \quad \forall s=\text { existing, } \\
& N_{s}-\gamma_{s}\left(1-Z_{s}\right) \geqslant 0 \quad \forall s=\text { existing, } \\
& \sum_{r=\text { trolley }} X_{r d p \mathrm{BTC}}-\beta_{\mathrm{BTC}} W_{\mathrm{BTC}} \leqslant 0 \quad \forall d, p .
\end{aligned}
$$

Variable restrictions:

$x_{\text {rdps }} \geqslant 0$,

$A_{s}, N_{s} \geqslant 0$ and integer,

$\omega_{s}, \lambda_{s}, \beta_{s}, \gamma_{s}, \theta_{s} \geqslant 0$

$\alpha_{s} \geqslant 1$,

$W_{s}, Y_{s}, Z_{s}=0,1$.

The subscripts denote:

$r$ route,

$d$ day (weekday, Saturday, or Sunday/holiday),

$p \quad$ service period (a.m. peak, p.m. peak, or all-day service),

$s$ transit center.

Model variables are:

$X_{r d p s} \quad$ the number of buses assigned to route $r$ on day $d$ for service period $p$ from transit center $s$;

$N_{s} \quad$ the total number of buses assigned to transit center $s$ (including spares);

$W_{\mathrm{BTC}} \quad 0$ if Boundary Road is not electrified between Hastings and Broadway (trolleys are then not assigned to Burnaby Transit Centre (BTC)); 1 if Boundary Road is electrified between Hastings and Broadway (trolleys are allocated to BTC). (Since this variable and its cost coefficient only apply to one transit center location, the subscript is given as "BTC", instead of $s$ );

$Z_{s} \quad 0$ if existing transit center $s$ remains open; 1 if existing transit center $s$ is shut down;

$A_{s} \quad$ the active number of buses assigned to transit center $s$;

$Y_{s} \quad 0$ if a transit center in candidate location $s$ is not opened; 1 if a transit center in candidate location $s$ is opened. 
Model parameters include the following:

\begin{tabular}{|c|c|}
\hline$C_{r d p s}$ & $\begin{array}{l}\text { the annual deadheading cost to operate one bus on route } r \text { on day } d \text { for service } \\
\text { period } p \text { from transit center } s \text {; }\end{array}$ \\
\hline$V_{s}$ & annualized per bus capital costs to construct a candidate transit center at site $s$; \\
\hline$F_{\mathrm{BTC}}$ & $\begin{array}{l}\text { annualized capital costs to electrify Boundary Road between Hastings Street and } \\
\text { Broadway so that trolleys could be allocated to the BTC; }\end{array}$ \\
\hline$R_{s}$ & annualized "salvage value" obtained, should existing transit center $s$ be eliminated; \\
\hline$D_{r d p}$ & number of buses required (demand) for route $r$ on day $d$ during service period $p$; \\
\hline$\alpha$ & $\begin{array}{l}\text { spare factor used to augment the number of active buses to allow for vehicle } \\
\text { breakdowns, major scheduled maintenance, or accidents; }\end{array}$ \\
\hline$\omega_{s}$ & maximum allowed size of candidate transit center $s$ \\
\hline$\lambda_{s}$ & minimum allowed size of candidate transit center $s$ \\
\hline$\beta_{s}$ & maximum allowed size of existing transit center $s$ \\
\hline
\end{tabular}

The objective function sums the total location/allocation costs. The first term in the objective function comprises the total vehicle deadhead cost. The second term calculates the total capital cost required to construct candidate transit centers, while the third term is used to assign a capital cost should the BTC be modified to accept trolley buses. The last term considers the salvage value that would accrue to the transit system, should an existing transit center be closed.

The first set of constraints ensures that the total demand for buses (in terms of specific routeday-service period combinations) is satisfied. The second type of constraints sums the total number of buses assigned to a specific transit center and ensures that it does not exceed $A_{s}$, the active number of buses allocated to a transit center $s$. Since a bus operating in the a.m. peak can also operate in the p.m. peak, a garage's active number of buses is the maximum of either the allday plus a.m. peak runs, or the all-day plus p.m. peak runs. We note that this summation is done over the three different day periods considered (weekday, Saturday, and Sunday/holiday).

The next set of constraints augments the active number of buses by a spare factor, $\alpha$, to give the total number of buses assigned to a transit center, $N_{s}$. The sizes of the candidate transit centers were forced to fall within pre-assigned capacity bounds. This reflected the judgments of transit staff concerning the appropriate values for minimum and maximum sizes of transit centers. The fourth and fifth types of constraints exhibit this. The next two categories of constraints perform a similar function for existing transit centers. The final constraint set ensures that, if trolleys are assigned to the BTC (and the concomitant capital charge is incurred), the total allocation cannot exceed the transit center's capacity.

The mathematical program contained roughly 12,000 variables (31 integer) and over 2000 constraints. A state-of-the-art commercial MIP package, CPLEX 2.0, was employed to solve this MIP problem. All model runs were performed at The University of British Columbia on a HP 9000 series 700 model 730 workstation. This workstation employed a $66 \mathrm{MHz}$ PA-RISC processor supporting the HP-UX UNIX operating system. Solution times to optimality were in the neighborhood of 5-7 min. Table 2 presents the optimal location and size of transit centers for the VRTS in the "base case" model. The total number of buses allocated in the current and optimal solutions may not necessarily be equivalent due to the "rounding up" that could occur when $A_{s}$ is 
Table 2

MIP model results

\begin{tabular}{llcc}
\hline Site number & Location & Current allocation & Optimal allocation \\
\hline E1 & North Vancouver & 78 & 60 \\
E2 & Port Coquitlam & 114 & 114 \\
E3 & Surrey & 132 & 123 \\
E4 & Burnaby & 166 & 160 \\
E5 & Oakridge & 410 & 350 \\
C1 & BCRTC & - & 50 \\
C2 & Cloverdale & - & 0 \\
C3 & Lougheed Park \& Ride & - & 0 \\
C4 & Richmond & - & 0 \\
C5 & Main \& Terminal & - & 50 \\
\hline
\end{tabular}

augmented by $\alpha$ to produce $N_{s}$ (note that $A_{s}$ and $N_{s}$ must take on integer values). Table 3 illustrates the costs associated with the optimal solution. Besides the optimal solution to the "base case" model, researchers were also interested in solutions for additional transit planning scenarios (for example, incorporating an additional rapid transit line, deleting an existing facility, or increasing the minimum allowable transit center size).

It is apparent that the optimal solution calls for a substantial shift in the transit system's location/allocation scheme. While all existing transit centers are retained, however, their capacities are now strictly obeyed. Two new facilities are constructed: one near the British Columbia Rapid Transit Corporation (BCRTC) facility in New Westminster, and the other near the Main \& Terminal intersection in Vancouver proper.

Annual savings of over $\$ 560,000$ are produced by the optimal solution. This reflects a drop of $3.77 \%$ in overall transit center costs. However, the optimal solution did not force all buses of a given route to be assigned from the same transit center. This occurred for 21 of the 110 routes in the transit network, representing just under $29 \%$ of the entire VRTS fleet.

This splitting of a route between alternative transit center locations represented a cause of concern for transit personnel. Representatives from the scheduling department and drivers alike felt a certain uneasiness, knowing that some buses of a specific route were allocated from one transit center, while the remaining portion of the route's buses were assigned from a different one. The operational and managerial inconvenience associated with such a solution ought not to be treated lightly.

An initial effort was made to develop an optimal MIP solution which would force all buses of a given route to be dispatched from the same transit center. The mathematical program was modified with the inclusion of the following parameter and variable, respectively:

Table 3

Breakdown of costs

\begin{tabular}{llr}
\hline Cost category & Current allocation $(\$)$ & Optimal allocation $(\$)$ \\
\hline Deadhead costs & $14,885,587$ & $13,288,690$ \\
Capital costs & 0 & $1,035,400$ \\
Total costs & $14,885,587$ & $14,324,090$ \\
\hline
\end{tabular}


$D_{r} \quad$ the number of buses required for route $r$;

$B_{r s} \quad 1$ if all of the buses of route $r$ are allocated from transit center $s ; 0$ if none of the buses of route $r$ are allocated from transit center $s$.

The $D_{r}$ parameter was obtained as the sum of a route's all-day requirements plus the maximum of either a.m. peak or p.m. peak needs. Since weekday requirements tend to dominate those of the weekend, the value for $D_{r}$ was usually obtained from weekday totals.

The following constraints were then included in the mathematical program:

$$
\begin{aligned}
& \sum_{d} \sum_{p} X_{r d p s} \leqslant D_{r} B_{r s} \quad \forall r, s, \\
& \sum_{s} B_{r s}=1 \quad \forall r .
\end{aligned}
$$

If $B_{r s}$ is equal to 1, then it can be seen from the first of these constraints that the total number of buses of route $r$ assigned to transit center $s$ could not exceed $D_{r}$, the requirements of the particular route. If $B_{r s}$ takes on the value of 0 , then $n o$ bus of route $r$ can be assigned to transit center $s$. Since $B_{r s}$ is a binary integer variable and its sum, over all $s$, is forced to equal 1 , we know that one and only one transit center will receive the allocation of buses of route $r$. The disadvantage with such an approach is the substantial increase in binary integer variables. The transit system under study contains 12 trolley routes (which can be assigned to any of three transit centers) and 98 diesel routes (which could be assigned to any of 10 transit centers). This represents an increase of 1016 binary integer variables in the model $((12 * 3)+(98 * 10))$.

The optimizer failed to obtain so much as an integer feasible solution after running for over $2.5 \mathrm{hrs}$ (real time), before the process was aborted. Based on our experience with earlier models, our optimizer took at most $7 \mathrm{~min}$ to find the best integer solution. The failure to determine an integer feasible solution within such an exceptionally long duration almost begs the creation of a heuristic procedure to resolve the splits in the MIP transit center problem. This, then, is the subject of the following section.

\section{Description of the heuristic procedure}

A crucial feature of the MIP optimal solutions is that all transit centers are within minimum/ maximum limits, but some routes are split. In order to assign all buses of a route to a unique transit center and still comply with size limitations, the proposed heuristic procedure follows a two-stage process. In the first stage, all split routes are assigned to a unique transit center with a temporary blind eye to transit center capacity violations. In the second stage, routes are switched to other transit centers so that garage capacity limitations are strictly observed. Fig. 2 depicts the various details of this heuristic procedure.

For each route split between transit centers, one calculates the total annual deadhead costs (TDHCs) of dispatching all buses of the route from each of the alternative transit centers. For 


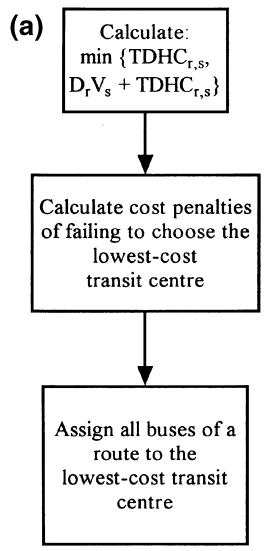

(b)

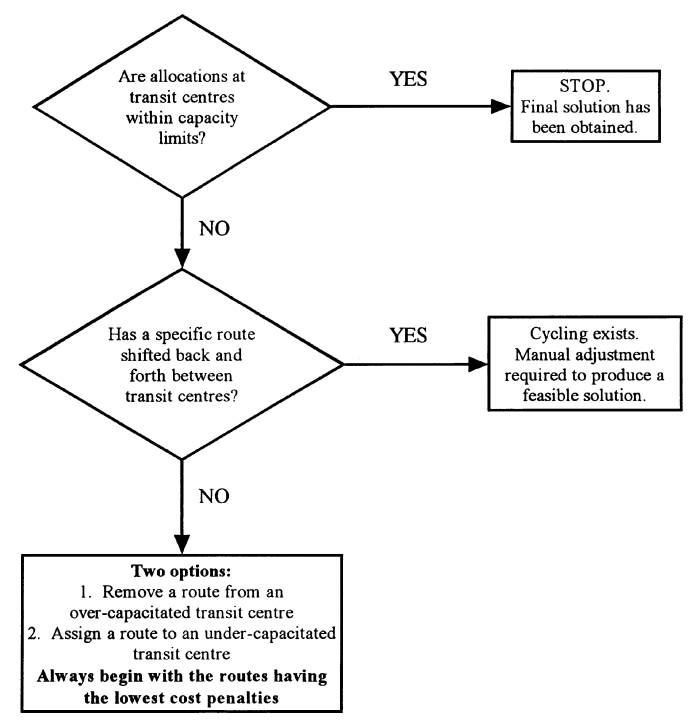

Fig. 2. Split resolution heuristic procedure. For each split route: (a) unique assignment; (b) capacity enforcement.

candidate facilities, we must also consider the annualized capital costs of building sufficient space to house the buses of the route. As a result, the following values are calculated (note that $V_{s}$ is the annualized per bus capital cost to construct a candidate transit center at site $s$ ):

- existing transit centers: $\mathrm{TDHC}_{r, s}$;

- candidate transit centers: $\mathrm{D}_{r} \mathrm{~V}_{s}+\mathrm{TDHC}_{r, s}$.

It becomes quite obvious that a split route is divided between a lower-cost transit center and a higher-cost transit center (or transit centers, if more than two garages are involved in the split). In the first stage of the heuristic procedure, we calculate the minimum of $\left\{\mathrm{TDHC}_{r, s}, \mathrm{D}_{r} \mathrm{~V}_{s}+\right.$ $\left.\mathrm{TDHC}_{r, s}\right\}$ for each split route. We also calculate the cost penalties that would accrue if any of the higher-cost transit centers were chosen. Then, we assign all the buses of the rejoined route to the lower-cost transit center. As an aside, we note that candidate transit centers will often exhibit a 
lower total annual deadhead cost for a split route; however, the concomitant capital costs of facility construction can outweigh the deadhead cost savings. After this first stage has been completed, all split routes have been rejoined. However, violations of transit center capacity limitations will undoubtedly occur.

The second part of the heuristic procedure seeks to restore compliance with capacity restrictions. One can begin by examining those transit centers that are above their capacity limits. We switch rejoined routes back to the higher-cost transit center, the ones with the lowest cost penalty first, until we are within capacity at each of these transit centers. Then, transit centers below the minimum capacity level are analyzed. We now assign the split routes previously assigned (and removed in the first stage of the heuristic procedure) which give the lowest cost penalty, continuing until the constraint violations are eliminated. At this point, all transit centers will meet the minimum capacity constraint.

The final solution obtained from using this heuristic procedure will assign the buses of each and every route to a unique transit center, plus capacities will be observed. One usually finds that a route has been split between an existing transit center and candidate facility. The former transit centers are usually above capacity after the first stage of the procedure, while the candidate transit centers (due to the relatively high cost of construction) fall below their minimum allowable levels. As a result, removing a route from an over-capacitated existing transit center and assigning it to a candidate transit center provides a "double-edged" effect.

There is, nonetheless, the possibility of "cycling" in this procedure. Suppose there are two existing transit centers, both exceeding their upper capacity restrictions. Taking a route from one of these transit centers and allocating it to the other may cause the first transit center to be under its maximum size limit. However, the other transit center will definitely continue to be above capacity. Switching the route back to the first transit center will again result in over-capacity problems for at least one of the transit centers. Thus, this route will continue to be bounced "back and forth" between alternative transit centers, with no feasible solution resulting. The same situation could occur if two candidate facilities, both under their minimum allowable size limits, were "battling" for a given route. Allocating the route to one of the transit centers may satisfy the minimum level for that transit center, but the other one would continue to violate the model constraints.

In the first situation, one manually removes the route in consideration from the pair of over-capacitated transit centers (and assigns it to the transit center which provides the lowest added cost and which is in no danger of maximum capacity violations). For the pair of transit centers under minimum allowable limits, one manually assigns a route from another garage, where the removal does not cause a constraint violation and which does this at lowest added cost.

Nonetheless, such problems are rare! (In fact, through all the model runs we performed in order to evaluate our heuristic procedure, we can only recall this situation occurring once.) On a practical level, there is unlikely to be an optimal MIP solution in which there would be two nearby transit centers at minimal occupancy levels. Even tiny cost differences should drive one transit center out of the optimal solution. Furthermore, the existing route and transit center network of the VRTS have been created in such a way that existing garages are not competing for the same route in the MIP optimal solutions. There is a relatively large distance between existing transit centers. 


\section{Results}

The proposed heuristic procedure was initially used on the original MIP "base case" optimal solution. Recall that in this instance, 21 routes were split, accounting for almost $29 \%$ of the VRTS bus fleet. As outlined in Section 4, the first step of the heuristic procedure assigns all buses of a route to a unique transit center, but can cause capacity violations. Table 4 indicates the total transit center allocations after the heuristic procedure's first stage. It also lists the total allocations after the second step has been implemented (the final solution). As an aside, we note that the total number of buses allocated in the first stage and final solution of the heuristic procedure may not necessarily be equivalent due to the manner in which $A_{s}$, the active number of buses assigned to transit center $s$, is determined. This value is the maximum of either all-day plus a.m. peak runs, or all-day plus p.m. peak runs. Suppose one combines, in a single transit center, a route dominated by a.m. peak runs with a route having mostly p.m. peak requirements. The total number of buses required in the transit center may not simply be the sum of the number of buses required for each route.

After the first step of the procedure, the two candidate transit centers, BCRTC and Main \& Terminal, both violate their minimum allowable sizes (the latter transit center has a particularly small allocation). The North Vancouver transit center also falls under its minimum allowable limit, while the Burnaby and Oakridge transit centers have allocations in excess of their capacity.

After routes were switched in the second stage of the heuristic procedure, the allocations at all transit centers fall within capacity limitations. Of particular importance is that the heuristic solution calls for additional allocations at candidate transit centers (Table 2 showed that under the MIP optimal solution, 50 buses were assigned to each candidate transit center).

Table 5 lists the cost of the heuristic solution. It exceeds the MIP optimal solution by approximately $1.06 \%$. More than half of the added cost is due to the extra capital costs incurred as the size of the candidate transit centers are increased. The total cost for the heuristic solution, however, still falls well under the current costs incurred by the VRTS under the prevailing location/allocation scheme. Our new solution represents an annual savings of over $\$ 400,000$, a reduction in costs of roughly $2.75 \%$. Transit company officials indicated that this constituted a rather substantial savings.

In order to examine the behavior of the heuristic procedure under a variety of starting conditions, it was used to generate solutions for other model runs. Table 6 provides the details on four additional model runs. The original MIP optimal solution is included for the sake of comparison.

Table 4

Transit centre sizes (Heuristic solution)

\begin{tabular}{llcc}
\hline Site number & Location & First stage & Final solution \\
\hline E1 & North Vancouver & 42 & 55 \\
E2 & Port Coquitlam & 121 & 101 \\
E3 & Surrey & 132 & 131 \\
E4 & Burnaby & 164 & 158 \\
E5 & Oakridge & 404 & 350 \\
C1 & BCRTC & 30 & 58 \\
C5 & Main \& Terminal & 13 & 51 \\
\hline
\end{tabular}


Table 5

Breakdown of costs (Heuristic solution)

\begin{tabular}{lcc}
\hline Cost category & MIP optimal solution $(\$)$ & Heuristic solution \\
\hline Deadhead costs & $13,288,690$ & $\$ 13,356,150$ \\
Capital costs & $1,035,400$ & $\$ 1,119,444$ \\
Total costs & $14,324,090$ & $\$ 14,475,594$ \\
Difference in total costs & & $\$ 151,504$ \\
Percentage difference & & $1.06 \%$ \\
\hline
\end{tabular}

Table 6

Costs of heuristic solution (Additional model runs)

\begin{tabular}{llllll}
\hline & $\begin{array}{l}\text { Original } \\
\text { optimal } \\
\text { solution }\end{array}$ & $\begin{array}{l}\text { "Minimum } \\
75 \text { " }\end{array}$ & $\begin{array}{l}\text { "Minimum } \\
100 "\end{array}$ & $\begin{array}{l}\text { Eliminate } \\
\text { Oakridge }\end{array}$ & $\begin{array}{l}\text { Increase } \\
\text { Burnaby } \\
\text { capacity }\end{array}$ \\
\hline Number of split routes & 21 & 18 & 16 & 28 & 20 \\
Percentage of bus fleet affected & $28.7 \%$ & $23.1 \%$ & $20.6 \%$ & $33.4 \%$ & $22.4 \%$ \\
MIP optimal solution costs & $\$ 14,324,090$ & $\$ 14,437,007$ & $\$ 14,501,487$ & $\$ 15,545,526$ & $\$ 13,872,106$ \\
Heuristic solution cost & $\$ 14,475,594$ & $\$ 14,698,532$ & $\$ 14,705,746$ & $\$ 15,829,165$ & $\$ 14,018,975$ \\
Difference in total costs & $\$ 151,504$ & $\$ 261,525$ & $\$ 204,259$ & $\$ 283,639$ & $\$ 146,869$ \\
Percentage difference & $1.06 \%$ & $1.81 \%$ & $1.41 \%$ & $1.82 \%$ & $1.06 \%$ \\
\hline
\end{tabular}

The "minimum 75" and "minimum 100" scenarios increased the minimum allowable size for a candidate facility to the indicated amount. The Oakridge transit center (the largest in the VRTS network) was forced to close in the "eliminate Oakridge" option, while the "increase Burnaby capacity" augmented the size of the Burnaby facility by the use of adjoining lands.

Table 6 also provides the number of split routes and percentage of bus fleet affected in each model run. It gives the costs of the heuristic solution, and the comparison of the costs (on an absolute and percentage level) to those arising in the MIP solution. The heuristic procedure performs well in each of the model runs we examined. This would suggest that our procedure can determine a reasonably good solution given a variety of starting conditions. We note that transit planners observed that the "minimum 100" model run represented a more realistic scenario for transit center construction. This larger size allows the company to spread out the tremendous effort of building a transit center (sub-contracting negotiations, zoning regulations, etc.) over more buses. The heuristic solution for this scenario is $1.41 \%$ higher than the corresponding MIP solution. Annual savings of $\$ 180,000$ occur between this heuristic solution and the current location/allocation scheme.

\section{Summary and conclusions}

Using mathematical programming to obtain the optimal number, size and location of transit centers is a practical and cost-effective approach. However, to address the concern over split routes, this paper has presented a simple heuristic procedure to force all the buses on a route to be 
allocated to a single transit center. Capacity limitations have been strictly observed. Cost comparisons with the MIP solutions indicate that the heuristic procedure performs well. It is the responsibility of the management to decide whether the effect of simpler personnel scheduling and easier management control justifies the reduction in savings incurred.

Cost comparisons were made between a heuristic solution in which no splitting was allowed and an MIP solution in which splitting occurred. The cost of the optimal solution for the case in which no splitting is allowed will fall somewhere between the two above-mentioned costs. If one could obtain the cost of the optimal solution (our optimizer was unable to solve this problem within a reasonable time), then it would be quite interesting to compare these with the cost arising from use of the heuristic procedure.

\section{Acknowledgements}

For their extensive support during the original project, the authors wish to thank the following employees of BC Transit: Robert N. Tribe, formerly Vice-President (Capital Projects and Engineering); W.H. (Bill) Green, Schedule Supervisor and Technical Assistant; Garry D. Andrews, Project Manager.

\section{References}

Cooper, L., 1963. Location-allocation problems. Operations Research 11, 331-343.

Fleischmann, B., Paraschis, J.N., 1988. Solving a large scale districting problem: a case report. Computers and Operations Research 15, 521-533.

Hess, S.W., Samuels, S.A., 1971. Experiences with a sales districting model: criteria and implementation. Management Science 18, 41-54.

Hojati, M., 1996. Optimal political districting. Computers and Operations Research 23, 1147-1161.

Khumawala, B.M., 1974. An efficient heuristic procedure for the capacitated warehouse location problem. Naval Research Logistics Quarterly 21, 609-623.

Love, R.F., Morris, J.G., Wesolowsky, G.O., 1988. Facilities Location: Models and Methods. North-Holland, New York.

Maze, T.H., Khasnabis, S., Kutsal, M.D., 1982. Optimization methodology for bus garage locations. Transportation Engineering Journal of ASCE 108, 550-569.

Maze, T.H., Khasnabis, S., Kutsal, M.D., 1983. Application of a bus garage location and sizing optimization. Transportation Research 17A, 65-72.

Uyeno, D.H., Willoughby, K.A., 1995. Transit centre location-allocation decisions. Transportation Research 29A, 263-272.

Willoughby, K.A., 1993. BUBLS: a mixed integer program for transit centre location in the lower mainland. Masters of Science Thesis, The University of British Columbia. 